Original Research Paper

\title{
Clinical Analysis of Pulmonary Mycobacterium kansasii Disease
}

\author{
Yoshihiro Kobashi, Masaaki Abe, Daisuke Yoshioka, Shigeki Kato and Toru Oga \\ Department of Respiratory Medicine, Kawasaki Medical School, 577 Matsushima, Kurashiki, Japan
}

\author{
Article history \\ Received: 08-10-2019 \\ Revised: $30-12-2019$ \\ Accepted: 10-01-2020 \\ Corresponding Author: \\ Yoshihiro Kobashi \\ Department of Respiratory \\ Medicine, Kawasaki Medical \\ School, 577 Matsushima, \\ Kurashiki, Japan \\ Tel: +81-86-462-1111 \\ Fax: +81-86-464-1041 \\ Email: yoshihiro@med.kawasaki-m.ac.jp
}

\begin{abstract}
To investigate the clinical aspects of pulmonary Mycobacterium kansasii (M. kansasii) disease. In 25 patients who satisfied the diagnostic criteria of the American Thoracic Society, we retrospectively evaluated the background, diagnostic methods, laboratory findings, radiological findings, treatment and prognosis. The median age was 61 years old (20 males, 5 females). Nine patients (36\%) had no underlying diseases. The definite diagnosis was obtained by expectorated sputum in 10 cases, bronchoscopy in 12, video-assisted thoracic surgery in 2 and CT-guided lung biopsy in 1 . The positive response rate for QuantiFERON (QFT) was 27\%. Radiological changes in pulmonary $M$. kansasii disease were frequently shown in the upper lobe and bilateral lung fields, but the extent of the lesions was limited within ten segments of the lung. Infiltration shadows or centrilobular small nodules with bronchiectasis, as well as cavities, were recognized in over half of all patients. Combined chemotherapy was given to 16 of 25 patients with a favorable effect in 15 . The remaining 9 patients consisted of 2 patients who required surgical treatment, one who died of other complications and six with no treatment because of hospital transfer. Patients with pulmonary $M$. kansasii disease may have radiological findings without cavitary disease described in previous studies. Therefore it is better to perform a bronchoscopic examination to make an accurate diagnosis and do smear and culture examinations for acid-fast bacilli.
\end{abstract}

Keywords: Pulmonary Mycobacterium kansasii (M. kansasii) Disease, Bronchoscopic Examination, QFT

\section{Introduction}

Mycobacterium kansasii (M. kansasii) is the third most common nontuberculous mycobacterial (NTM) infectious disease following M. avium and M. intracellulare in Japan (Morimoto et al., 2014; Namkoong et al., 2016). The incidence of this disease was stable in Japan (Namkoong et al., 2016) and the ratio of this disease consisted of small population of pulmonary disease in the United States of America (Spaulding et al., 2017).

In 2007, the American Thoracic Society (ATS) proposed both clinical and microbiological criteria be required for the diagnosis of pulmonary NTM disease. Microbiological results require positive culture from at least two separate expectorated samples or positive culture from one bronchial wash or alveolar lavage, transbronchial or other lung biopsy with mycobacterial histologic features and a positive culture for NTM
(Griffith et al., 2007). The Japanese Society for Tuberculosis (JST) also proposed similar criteria in 2008 (GDPNMD, 2008).

Regarding the clinical characteristics of pulmonary M. kansasii disease, it has been previously reported that most patients are male with underlying respiratory diseases such as Chronic Obstructive Pulmonary Disease (COPD) and that radiological findings are similar to pulmonary tuberculosis with a thin-wall cavity (Christensen et al., 1978). To further clarify the clinical characteristics of pulmonary M. kansasii disease, we retrospectively investigated its clinical and microbiological characteristics.

\section{Materials and Methods}

Twenty-five patients in whom pulmonary M. kansasii disease was diagnosed in Kawasaki Medical School 
Hospital between January 2007 and December 2017 were retrospectively investigated in this study and all of them satisfied the diagnostic criteria of pulmonary NTM disease proposed by the ATS (Griffith et al., 2007). All patients enrolled in this study were retrospectively investigated with regard to the following clinical characteristics from the chart review: age, sex, underlying disease, detection methods, laboratory findings (inflammatory response such as leukocytosis, Erythrocyte Sedimentation Rate (ESR) and C-reactive Protein (CRP), nutritional conditions such as total protein or serum albumin, microbiological findings, radiological findings, treatment details and clinical effects and prognosis. Regarding the results of QuantiFERON (QuantiFERON Gold (QFT-G) test or QuantiFERON-TB Gold In-Tube (QFT-GIT) test), we collected from the chart review including the results that we performed according to the recommendations of the manufacturer (Cellestis Ltd., Australia) in 22 of 25 patients. The judgement was performed according to the guideline proposed by the Centers for Disease Control and prevention (CDC) for using QFT (Mazurek et al., 2010).

Concerning the microbiological examinations in the laboratory center of our hospital, clinical specimens such as expectorated sputum, bronchoscopy and tissue were directly examined by Ziehl-Neelsen staining. The specimens used for culture were digested and decontaminated by the sodium hydroxide method. The samples were inoculated onto slants of 1\% Ogawa egg medium and bacteria were identified and differentiated based on growth characteristics and conventional biochemical tests. Drug sensitivity tests for isolated $M$. kansasii were performed in 22 of 25 patients due to the retrospective study. Minimal Inhibitory Concentrations (MICs) for Isoniazid (INH), Rifampicin (RFP), Ethambutol (EB), streptomycin (SM), clarithromycin (CAM), Levofloxacin (LVFX) and Amikacin (AMK) were determined by microdilution based on the broth MIC NTM (Kyokuto Pharmaceutical Industrial Co. Ltd).

Regarding the radiological findings on admission, the portion and extension of the lesions were evaluated based on both chest radiographs and Computed Tomography (CT) findings. We counted the number of involved segments of the lung about the extension of the lesions. Thereafter, we classified into the fibrocavitary form or the nodular bronchiectatic form. If the findings did not belong to two categories, it was deemed unclassifiable (Moon et al., 2015). Finally, we reviewed the radiological findings such as cavities, infiltration shadows, centrilobular small nodules, solitary nodular shadows ( $\geqq 2 \mathrm{~cm}$ ), bronchiectasis and lymph node swelling.

This study was approved by the Ethical Committee of Kawasaki Medical School (No. 3155).

\section{Results}

The backgrounds of the 25 patients with pulmonary M. kansasii disease are shown in Table 1. Among the 25 patients (mean age: 57.4 years old, male/female 20/5), 16 had underlying diseases. Eight had respiratory underlying diseases including healed pulmonary tuberculosis or Chronic Obstructive Pulmonary Disease (COPD). Although we investigated HIV antibody for patients suspected of HIV infection such as lymphocytopenia as far as possible, there were no HIV (Human immunodeficiency virus) antibody positive patients in this study.

Table 1: Backgrounds of pulmonary $M$. kansasii disease patients

\begin{tabular}{ll}
\hline Age (Mean, range) & $57.4(28-85)$ \\
Sex (Male: Female) & $20: 5$ \\
Smoking history $(+)$ & $19(76 \%)$ \\
Alcohol abuse $(+)$ & $2(8 \%)$ \\
Underlying disease $(+)($ with repetition) & $16(64 \%)$ \\
Respiratory disease & $8(32 \%)$ \\
Healed pulmonary tuberculosis & 4 \\
COPD & 2 \\
Pneumoconiosis & 1 \\
NSIP & 1 \\
Bronchial asthma & 1 \\
Non-respiratory disease & $12(48 \%)$ \\
Gastrointestinal disease & 4 \\
Cerebroneurovascular disease & 3 \\
Diabetes mellitus & 2 \\
Chronic renal failure & 2 \\
Others & 4 \\
Underlying disease(-) & $9(36 \%)$ \\
\hline COPD: Chronic Obstrive
\end{tabular}

COPD: Chronic Obstructive Pulmonary Disease, NSIP: Nonspecific Interstitial Pneumonia

Table 2: Radiological findings of pulmonary $M$. kansasii disease

\begin{tabular}{lc}
\hline Distribution of lesions & \\
Right & $8(32 \%)$ \\
Left & $6(24 \%)$ \\
Bilateral & $11(44 \%)$ \\
Upper lobe dominant & $19(76 \%)$ \\
Lower/middle lobe dominant & $6(24 \%)$ \\
Extension of lesions (Number of involved segments) \\
$1-5$ & $13(52 \%)$ \\
$6-10$ & $11(44 \%)$ \\
$11-15$ & $1(4 \%)$ \\
Type of disease & \\
Fibrocavitary dominant & $15(60 \%)$ \\
Nodular-bronchiectatic dominant & $6(24 \%)$ \\
Unclassifiable & $4(16 \%)$ \\
Radiological findings (with repetition) & \\
Cavity & $16(64 \%)$ \\
Infiltration shadow & $17(68 \%)$ \\
Bronchiectatic change & $17(68 \%)$ \\
Small nodular shadow & $20(80 \%)$ \\
Solitary nodular shadow $(\geqq 2 \mathrm{~cm})$ & $3(12 \%)$ \\
Lymph node swelling $(\geqq 1 \mathrm{~cm})$ & $1(4 \%)$ \\
\hline
\end{tabular}


Reasons for investigation were clinical symptoms such as cough, sputum and fever in 15 patients $(60 \%)$, while the remaining 10 patients were diagnosed during periodical radiological examinations or during the follow-up period for other underlying diseases (Table 2).

The laboratory findings on the first visit to our hospital were mild elevation of the ESR and CRP without leukocytosis in most patients. However, nutritional conditions such as total protein or serum albumin ranged within normal limitations in most patients. The QFT test (QFT-G test: 12 patients, QFT-IT test: 10 patients) showed positive responses in 6 of 22 patients (27\%), including one patient with healed pulmonary tuberculosis. One patient showed an indeterminate response. In 15 patients the QFT test was negative, including three patients with healed pulmonary tuberculosis.

A definite diagnosis was obtained from a sputum specimen in ten patients (40\%) (five patients had a past history of respiratory diseases), a local specimen using bronchoscopy (brushing, Bronchoalveolar Lavage Fluid (BALF) or Transbronchial Lung Biopsy (TBLB)) in twelve (48\%), surgical specimens using Video-Assisted Thoracoscopic Surgery (VATS) in two (8\%) and local specimen using CT-guided lung biopsy in one (4\%), respectively. Concerning the microbiological findings, smear-positive and culture-positive for acid-fast bacilli accounted for 21 patients (84\%) (sputum 7 patients, BALF 11, VATS 2 and CT-guided lung biopsy 1) and smear-negative, culture-positive for acid-fast bacilli accounted for 4 patients (16\%) (sputum 3 and BALF 1). Drug sensitivity tests for antimicrobial agents were performed for 22 of 25 patients with pulmonary $M$. kansasii disease. The results were comparatively good for RFP (MIC $\leqq 2 \mu \mathrm{g} / \mathrm{mL}$ in all patients).

Lesions revealed by chest $\mathrm{CT}$ were most frequently localized in the upper lobe. The extension of these lesions was limited within ten segments of the lung in most patients. Based on the radiological findings in all 25 patients, $15(60 \%)$ patients manifested the fibrocavitary form dominant, $6(24 \%)$ had the nodular bronchiectatic form dominant and $4(16 \%)$ exhibited an unclassifiable form. The characteristic radiological findings were centrilobular small nodules with bronchiectatic changes (Fig. 1) and infiltration shadows in over half of all patients with pulmonary $M$. kansasii disease. Cavities were recognized in $64 \%$ and three of 25 patients $(12 \%)$ showed solitary nodular lesions $(\geqq 2 \mathrm{~cm})$ which required differentiation from lung cancer (Fig. 2). On the other hand, mediastinal lymph node swelling was recognized in one patient (4\%).

Combined chemotherapy using INH, RFP and EB was administered to 16 patients. Among these patients, 15 (94\%) showed a good clinical response, but because one young patient could not continue the medical treatment due to adverse events and one was required differentiation of lung cancer, surgical resection was performed. Finally, surgical treatment was performed for two patients, including one patient with side effects and there were no recurrences without combined chemotherapy after resection. Because one patient died of complications (pneumonia, pneumothorax and heart failure) before a definite diagnosis was made, we could not administer any medical treatment. The remaining eight patients were not receiving treatment and lacked information regarding treatment due to hospital transfer.
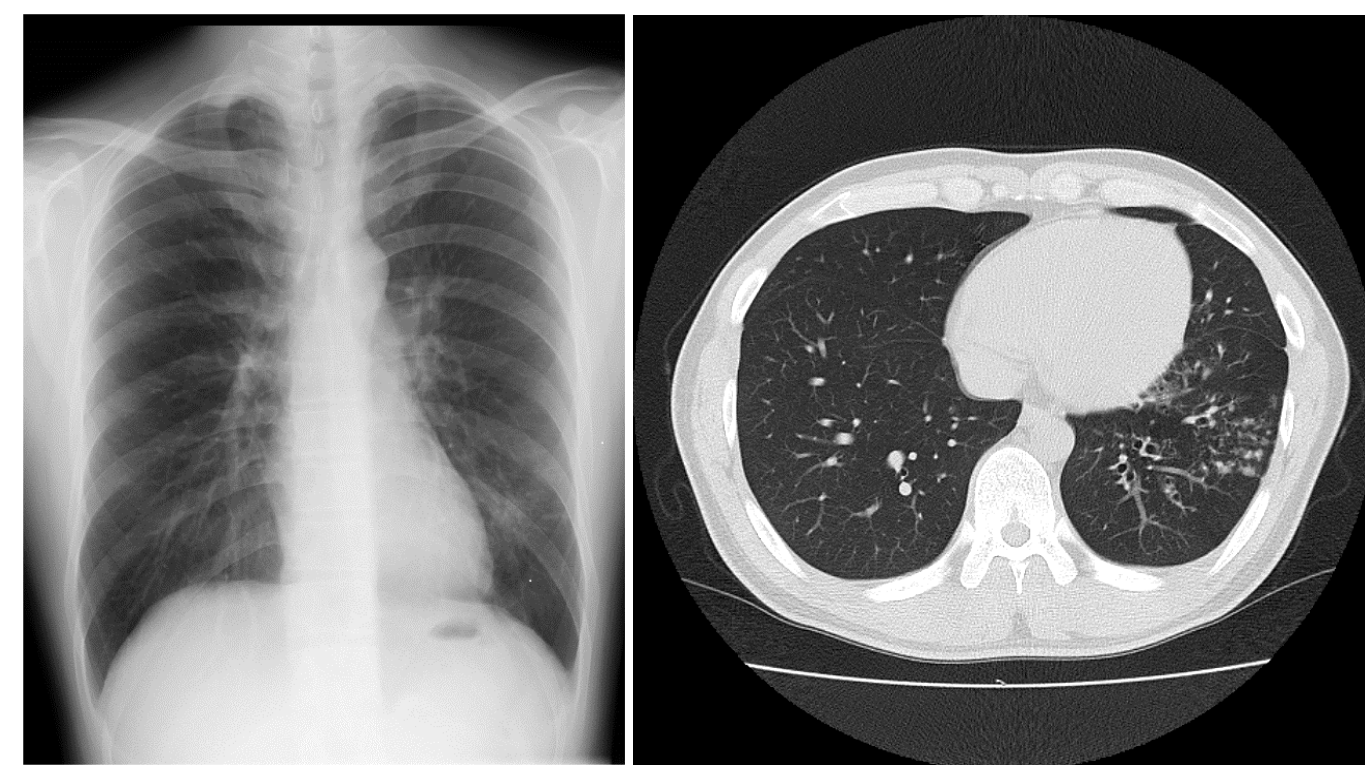

Fig. 1: A 32-year-old man showed centrilobular small nodules with bronchiectatic changes in the left lower lobe 

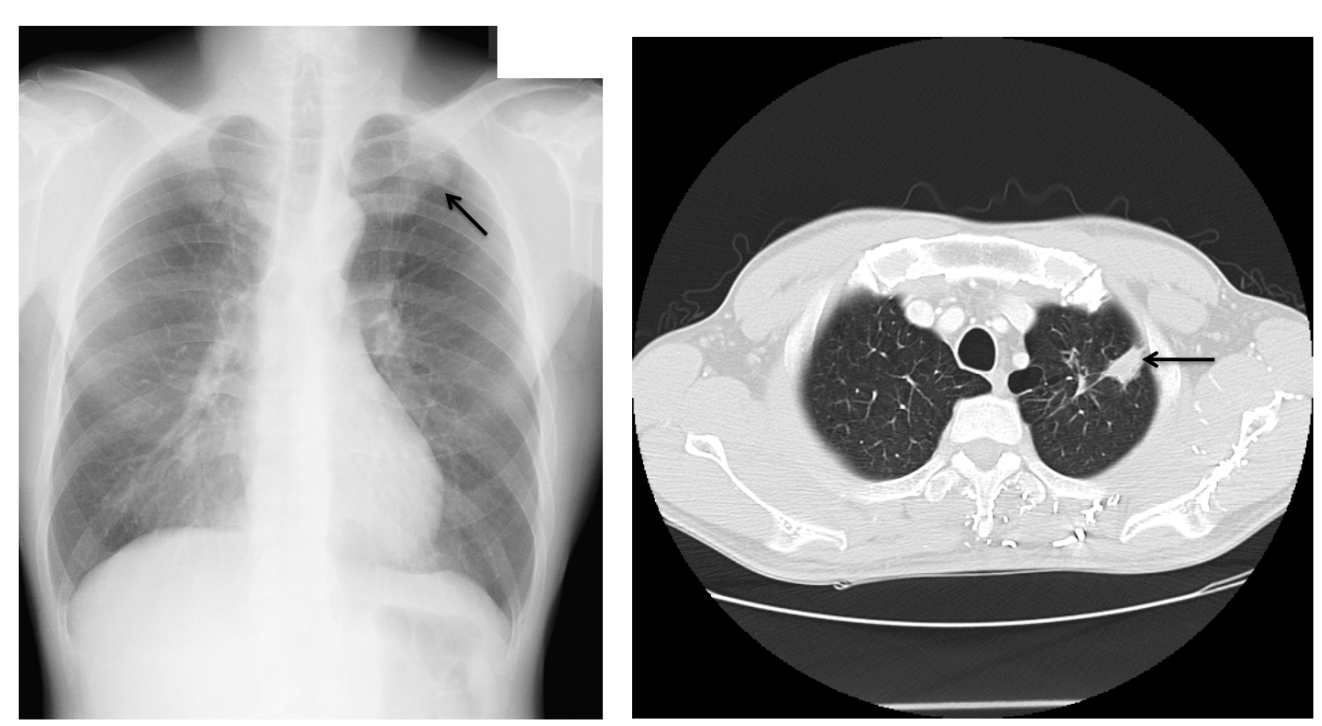

Fig. 2: A 46-year-old man showed nodular lesions $(>2 \mathrm{~cm})$ in the left upper lobe (arrow) which required differentiation from lung cancer

\section{Discussion}

Although COPD and healed pulmonary tuberculosis were significant predictors of pulmonary $M$. kansasii disease, underlying respiratory disease in the present patients occurred less frequently. The reasons for this were as follows; (1) four female patients had no smoking history and two other younger patients had a short-term smoking history in this study, (2) because this study was retrospective, all data from patients were drawn from charts and pulmonary function testing was only performed for some patients. Therefore, we could not recognize whether patients with a smoking history satisfied the diagnostic criteria of COPD and so did not include these patients as "patients with pulmonary M. kansasii disease with a past history of COPD" in this study.

Sixty percent of patients in the present study complained of clinical symptoms such as productive cough and $40 \%$ were detected from abnormal radiological findings on periodical chest radiographs or chest radiographs during the follow-up period for other underlying diseases. This finding may have resulted from the active performance of bronchoscopy. In fact, most of the present cases with no clinical symptoms were diagnosed by using bronchoscopic specimens obtained from brushing or BALF. A new diagnostic criterion for pulmonary NTM disease proposed by the ATS and JST (Griffith et al., 2007; GDPNMD, 2008) includes a positive culture result from local specimens such as brushing or BALF. In previous reports, only $10 \sim 20 \%$ of all patients with pulmonary M. kansasii disease underwent bronchoscopy, so a definite diagnosis for most patients previously depended on several expectorated sputum specimens. Therefore, a long time was required to make the correct diagnosis (Park et al., 2010; Matveychuk et al., 2012). M. kansasii is considered to be the most virulent among NTMs; its sensitivity to antimicrobial agents is comparatively high and the prognosis is favorable with appropriate treatment. Therefore, early and correct diagnosis of pulmonary $M$. kansasii disease by bronchoscopy is clinically important.

The positive response rate for QFT (27\%) was higher than that of previous reports (Takahashi et al., 2012; Inoue et al., 2013; Sato et al., 2016) among the present cases of pulmonary M. kansasii disease. In this study, although one of four patients with healed pulmonary tuberculosis showed a positive QFT response, remaining three patients showed a negative response. Even if three patients with healed pulmonary tuberculosis were excluded, the positive response rate of QFT was almost same (5/19: 26\%). The reason of high QFT positive response rate may be geographical characteristic of isolated M. kansasii. Since M. kansasii has the same Mycobacterium tuberculosis (MTB)-specific protein antigen (CFP-10, ESAT-6) as included in the QFT kit, false-positive QFT responses for patients with pulmonary $M$. kansasii disease are suggested to occur to some extent.

Regarding the radiological findings related to pulmonary M. kansasii disease, CT findings can be summarized as follows: (1) the distribution of lesions was most frequent in the upper lobe and bilateral lung field, (2) the extent of lesions was limited within ten segments of the lung, (3) infiltration shadows or centrilobular small nodules with bronchiectasis were recognized in over half of all patients, (4) typical radiological findings such as cavities were recognized in $64 \%$ of all patients. The distribution of lesions was similar to that previously reported (Inoue et al., 2013; 
Sato et al., 2016) and only laterality was different from previous reports (right lung dominant). The propensity of pulmonary lesions to affect the upper lobe in pulmonary M. kansasii disease was similar to that of MTB. High oxygen tension in the apex helps M. kansasii to be preferentially localized. The incidence of cavity formation has been considered high in patients with pulmonary $M$. kansasii disease, varying from $70 \%$ to $83 \%$, as in previous reports from Japan (Takahashi et al., 2012; Sato et al., 2016). However, more recent studies reported incidence from 32 to 53\% (Park et al., 2010; Matveychuk et al., 2012). The present $64 \%$ was thus an intermediate percentage. This discrepancy in the incidence of cavity formation may be explained by recent improvements in the diagnostic methods used (bronchoscopy etc.) and microbiological isolation of the organism.

Regarding the treatment for pulmonary M. kansasii disease, the ATS and JTS recommend a regimen including INH, RFP and EB for a duration of 12 months after negative cultures, especially with a threedrug treatment regimen. We used INH, RFP and EB according to the ATS and JST for 16 patients. Fifteen of 16 patients completed the treatment for at least 12 months and with negative culture results. Finally, 15 patients showed a favorable clinical effect. In this study, susceptibility demonstrated comparatively good MIC for RFP in most patients. However, surgical treatment was required for two patients because of side effects due to medical treatment and the differentiation of lung cancer before confirming the correct diagnosis in this study. Although antimicrobial agents such as CAM or fluoroquinolones may be important and reasonable alternatives (Griffith et al., 2007) in patients who showed poor susceptibility for RFP, we have to consider the indication of surgical treatment for cases refractory to medical treatment.

There are several limitations to our study. First, because it was a retrospective study that used medical records for data collection, we cannot obtain the data of QFT or drug sensitivity test for all patients. Second, we only studied a small number of patients ( 25 patients) in a single tertiary hospital. We need to perform a large-scale prospective study in Japan. Third, although we investigated the results of HIV antibody for all patients in this study, there were no patients with HIV antibody positive response. Therefore, we cannot deny the influence of the immunosuppressive state due to HIV infection completely. Fourth, there was no molecular characterization of the isolates of $M$. kansasii strains and genotypes may vary with regard to clinical significance.

\section{Conclusion}

Because patients with pulmonary M. kansasii disease have recently shown variations in radiological manifestations resembling other pulmonary NTM disease or requiring the differentiation from lung cancer, it is difficult to distinguish pulmonary M. kansasii disease without characteristic findings such as cavity formation from other pulmonary NTM diseases. Therefore, we recommend obtaining appropriate local specimens using invasive diagnostic methods such as bronchoscopy and describe the importance of making a correct diagnosis and selecting an appropriate treatment.

\section{Acknowledgment}

The investigators would like to thank Dr. Daigo Tanimoto, Division of Radiological diagnosis, Kawasaki Medical School.

\section{Author's Contributions}

Yoshihiro Kobashi: Research plan and organized the study writing of the manuscript.

Masaaki Abe and Daisuke Yoshioka: Help of the data collection of this study.

Shigeki Kato: Help of the data collection of this study and check of this manuscript.

Toru Oga: Final check of this manuscript.

\section{Conflict of Interest}

There are no conflicts of interests regarding this manuscript.

\section{References}

Christensen, E.E., G.W. Dietz, C.H. Ahn, J.S. Chapman and R.C. Murry et al., 1978. Radiologic manifestations of pulmonary Mycobacterium kansasii infections. Am. J. Roentogenol., 131: 985-993. DOI: 10.2214/ajr.131.6.985

GDPNMD, 2008. Guideline for the diagnosis of pulmonary nontuberculous mycobacterial diseases- 2008. The nontuberculous mycobacteriosis control committee of the Japanese society for tuberculosis, the scientific assembly for infection and tuberculosis of the Japanese respiratory society. Kekkaku, 86: 37-39.

Griffith, D.E., T. Aksamit, B.A. Brown-Ellion, A. Catanzaro and C. Daley et al., 2007. An official ATS/IDSA statement: Diagnosis, treatment and prevention of nontuberculous mycobacterial diseases. Am. J. Respir Crit. Care Med., 175: 367-416. DOI: $10.1164 / \mathrm{rccm} .200604-571 \mathrm{ST}$

Inoue, K., M. Senoo, N. Nagayama, K. Masuda and H. Matsui et al., 2013. A comparison of chest radiographs between patients with pulmonary Mycobacterium kansasii infection and those with Mycobacterium tuberculosis infection in the initial stage of disease. Kekkaku, 88: 619-623. 
Matveychuk, A., L. Fuks, R. Priess, I. Hahim and D. Shitrit, 2012. Clinical and radiological features of Mycobacterium kansasii and other NTM infections. Resp. Med., 106: 1472-1477.

DOI: $10.1016 /$ j.rmed.2012.06.023

Mazurek, G.H., J. Jereb, A. Vernon, P. LoBue and S. Goldberg et al., 2010. Updated guidelines for using interferon Gamma Release Assays to detect Mycobacterium tuberculosis infection- United States. MMWR Recomm. Rep., 59: 1-25.

Moon, S.M., H.Y. Park, K. Jeon, S.Y. Kim and M.J. Chung et al., 2015. Clinical significance of Mycobacterium kansasii isolates from respiratory specimens. PloS ONE, 10: e0139621-e0139621. DOI: 10.1371 /journal.pone.0139621

Morimoto, K., K. Iwai, K. Uchimura, M. Okumura and T. Yoshiyama et al., 2014. A steady increase in nontuberculous mycobacteriosis mortality and estimated prevalence in Japan. Ann. Am. Thoracic Soc., 11: 1-8.

DOI: 10.1513/AnnalsATS.201303-067OC

Namkoong, H., A Kurashima, K. Morimoto, Y. Hoshino and $\mathrm{N}$ Hasegawa et al., 2016. Epidemiology of pulmonary nontuberculous mycobacterial disease, Japan. Emerg. Infect. Dis., 22: 1116-1117.

DOI: $10.3201 /$ eid2206.151086
Park, H.K., W.J. Koh, T.S. Shim and O.J. Kwon, 2010. Clinical characteristics and treatment outcomes of Mycobacterium kansasii lung disease in Korea. Yansei Med. J., 51: 552-556. DOI: $10.3349 / \mathrm{ymj} .2010 .51 .4 .552$

Sato, R., H. Nagai, H. Matsui, Y. Kawabe and K. Takeda et al., 2016. Interferon-gamma release assays in patients with Mycobacterium kansasii pulmonary infection: A retrospective survey. J. Infect., 72: 706-712.

DOI: $10.1016 /$ j.jinf.2016.03.011

Spaulding, A.B., Y.L. Lai, A.M. Zelazny, K.N. Oliver and S.S. Kadri et al., 2017. Geographic distribution of nontunberculous mycobacterial species identified among clinical isolates in the United States, 2009-2013. Ann. Am. Thorac Soc., 14: 1655-1661.

DOI: 10.1513/AnnalsATS.201611-860OC

Takahashi, M., H. Tsukamoto, T. Kawamura, Y. Mochizuki and M. Ouchi et al., 2012. Mycobacterium kansasii pulmonary infection: CT findings in 29 cases. Jpn. J. Radiol., 30: 398-406. DOI: $10.1007 / \mathrm{s} 11604-012-0061-\mathrm{z}$ 